\title{
Spectral Modification by Operant Conditioning of Cortical Theta Suppression in Rats
}

\author{
Mootaek Roh ${ }^{1,3}$, II-Sung Jang ${ }^{2,3}$, Kyoungho Suk ${ }^{1,3}$, Maan-Gee Lee ${ }^{1,3}$ \\ ${ }^{1}$ Department of Pharmacology, School of Medicine, Kyungpook National University, ${ }^{2}$ Department of Pharmacology, School of Dentistry, \\ Kyungpook National University, ${ }^{3}$ Brain Science and Engineering Institute, Kyungpook National University, Daegu, Korea
}

\begin{abstract}
Objective: Brain activity is known to be voluntarily controllable by neurofeedback, a kind of electroencephalographic (EEG) operant conditioning. Although its efficacy in clinical effects has been reported, it is yet to be uncovered whether or how a specific band activity is controllable. Here, we examined EEG spectral profiles along with conditioning training of a specific brain activity, theta band $(4-8 \mathrm{~Hz})$ amplitude, in rats.

Methods: During training, the experimental group received electrical stimulation to the medial forebrain bundle contingent to suppression of theta activity, while the control group received stimulation non-contingent to its own band activity.

Results: In the experimental group, theta activity gradually decreased within the training session, while there was an increase of theta activity in the control group. There was a significant difference in theta activity during the sessions between the two groups. The spectral theta peak, originally located at $7 \mathrm{~Hz}$, shifted further towards higher frequencies in the experimental group.

Conclusion: Our results showed that an operant conditioning technique could train rats to control their specific EEG activity indirectly, and it may be used as an animal model for studying how neuronal systems work in human neurofeedback.
\end{abstract}

KEY WORDS: Electroencephalography; Neurofeedback; Operant conditioning; Theta; Rats.

\section{INTRODUCTION}

Converging evidence supports the notion that various rhythmic activities are closely related to distinct brain functions. ${ }^{1-4)}$ The alpha rhythm in electroencephalography (EEG) is associated with cognitive performance in humans. ${ }^{1)}$ The hippocampal theta rhythm is related to learning in rats, ${ }^{5,6)}$ rabbits $^{7,8)}$ and humans. ${ }^{9)}$ As expected from these results, abnormalities in or loss of rhythmic activity is associated with functional deficits, ${ }^{10,11)}$ and restoring rhythmic activity restores function in rats. ${ }^{12)}$ Moreover, the enhancement of sensorimotor rhythms (12-20

Received: September 22, 2017 / Revised: October 25, 2017

Accepted: October 26, 2017

Address for correspondence: Maan-Gee Lee, MD, PhD

Lab for Control of Brain Activity, Department of Pharmacology,

School of Medicine, Kyungpook National University, Room 516,

Biomedical Science Building, 136-gil 90, Chilgokjungangdae-ro,

Buk-gu, Daegu 41405, Korea

Tel: +82-53-950-4231, E-mail: mglee@knu.ac.kr

ORCID: https://orcid.org/0000-0002-0172-1593
$\mathrm{Hz}$ ) by operant conditioning resulted in the elevation of the seizure resistance threshold against epileptogenic compounds. ${ }^{13)}$ These results strongly argue for the notion that changes in rhythmic activity are cardinal for adaptive brain functions.

Over the last several decades, a number of human studies examining voluntary control of rhythmic activity by training have been documented. ${ }^{14-20)}$ This self-regulation, also known as neurofeedback training, has been widely interpolated to patients with neuropsychiatric disorders, such as anxiety, ${ }^{21)}$ epilepsy, ${ }^{22,23)}$ attention deficit hyperactivity disorder, ${ }^{24,25)}$ addictive disorders, ${ }^{26,27)}$ post-traumatic stress disorder, ${ }^{28)}$ and depression. ${ }^{29)}$ Their therapeutic efficacy has been reported in most cases. Furthermore, it has been reported that healthy individuals could improve cognitive ${ }^{14,17,30-32)}$ or behavioral performance $^{16)}$ and surgical skills ${ }^{17)}$ by training.

Even though most studies have shown post-training task performance and training effects, it is unclear whether the

(c) This is an Open-Access article distributed under the terms of the Creative Commons Attribution Non-Commercial License (http://creativecommons.org/licenses/by-nc/4.0) which permits unrestricted non-commercial use, distribution, and reproduction in any medium, provided the original work is properly cited. 
neurofeedback of specific frequency bands can really produce specific band activity modulation. A detailed description of the variation of rhythmic activity or the whole spectrum is usually neglected, and spectral profiles during training are rarely reported. ${ }^{33)} \mathrm{A}$ few studies focused on quantifying band activity changes with neurofeedback training. ${ }^{20,32)}$

Neurofeedback has been considered operant conditioning of EEG band activity. One difference between neurofeedback and operant conditioning is that changes in brain activity alone are not consciously perceived, but behavioral changes, such as lever-pressing, are consciously perceived. In neurofeedback, there is a physical stimulus converted from brain activity used as an explicit response. Another difference is that there is no contingent reward in neurofeedback training in human studies, while there is an explicit contingent reward in operant conditioning. Reward is essential for operant conditioning and is known to be important in neurofeedback training. ${ }^{34)}$ In neurofeedback, verbal mentioning of beneficial effects is provided before training, while an actual reward, such as food, water, or brain stimulation, is given contingently to a specific behavior in operant conditioning. There is controversy about reward in neurofeedback. Beneficial effects in neurofeedback are presented only with sensory feedback and verbal descriptions of the beneficial effects of training. ${ }^{35,36)}$ Therefore, the reward in neurofeedback may be contingent to the subject's expectation of the beneficial effect, and the expectation itself may induce a physiological change, such as brain activity. ${ }^{37,38)}$ Additionally, beneficial effects may be due to the induced subjective state during neurofeedback training. ${ }^{39)}$

Considering a specific EEG activity as a kind of operandum, we questioned whether operant conditioning could modulate specific brain activity in a manner similar to reinforcement of voluntary behavior in traditional operant conditioning. To answer to this question, we used the intracranial self-stimulation (ICSS) paradigm ${ }^{40)}$ as a reward. ICSS is a widely used experimental method in many operant conditioning studies, ${ }^{41,42)}$ and animals learn how to behave to be rewarded. Here, we trained rats to suppress (instead of increase) their theta activity, as stimulation of the medial forebrain bundle (MFB) induces theta activity. ${ }^{43-45)}$ In this animal model, it was possible to exclude expectation bias, which has a compounding effect in human neurofeedback. ${ }^{37,38)}$ Here, we examined wheth- er the theta activity could be voluntarily modulated with MFB stimulation as the reward and whether activity of a specific frequency range could be modulated in rats. Lastly, the feasibility of the neurofeedback animal model using brain reward stimulation was addressed.

\section{METHODS}

\section{Animals and Ethics Statement}

Male Sprague-Dawley rats (Samtaco, Osan, Korea) weighing 300 to $350 \mathrm{~g}$ were housed in polycarbonate cages ( 25 [wide] $\times 40$ [deep] $\times 17$ [high] $\mathrm{cm}$ ) with ad libitum access to food and water. The light/dark cycle was automatically controlled to 12/12 hours (light on 07:0019:00), and a room temperature of $20-25^{\circ} \mathrm{C}$ was maintained. After implantation surgery, rats were housed individually in separate cages. All experiments were conducted in accordance with approved protocols and guidelines established by the Animal Care Committee of Kyungpook National University (approval No. KNU2009-28) and the National Institute of Health Guide for the Care and Use of Laboratory Animals.

\section{Surgery}

General anesthesia was carried out by intraperitoneal injection of a $2 \mathrm{ml} / \mathrm{kg}$ cocktail of $10 \mathrm{ml}$ ketamine hydrochloride $(50 \mathrm{mg} / \mathrm{ml}), 1.1 \mathrm{ml}$ xylazine hydrochloride $(23.32 \mathrm{mg} / \mathrm{ml})$, and $2.67 \mathrm{ml}$ saline. Anesthesia was confirmed by unresponsiveness to tail and toe pinches. Animals were then fixed in a stereotactic apparatus (David Kopf Instruments, Tujunga, CA, USA). A midline incision in the scalp was performed after subcutaneous injection of $2 \%$ lidocaine. The periosteum was removed. The surface of the skull was cleaned with $3 \%$ acetic acid. The skull position was adjusted to ensure the bregma and the lambda had the same vertical coordinates. Holes were carefully drilled, and gold-plated stainless steel screws $(\sim 1$ $\mathrm{mm}$ diameter) were implanted into the skull (frontal AP + $2 \mathrm{~mm}, \mathrm{~L} \pm 1.5 \mathrm{~mm}$ from the bregma; parietal AP $-6 \mathrm{~mm}$, $\mathrm{L} \pm 5 \mathrm{~mm}$ from the bregma; and occipital $\mathrm{AP}-3 \mathrm{~mm}, \mathrm{~L} \pm 2$ $\mathrm{mm}$ from lambda). Holes $2 \mathrm{~mm}$ in diameter were bilaterally drilled into the skull (AP $-2.3 \mathrm{~mm}, \mathrm{~L} \pm 1.8 \mathrm{~mm}$ ), and the dura mater was carefully removed. Formvar-coated \#0 insect-pin electrodes were vertically inserted down to the MFB ( $-8.6 \mathrm{~mm}$ below the dura mater). Finally, connecting pins for EEG recording and MFB stimulation 
were assembled in a package and fixed with dental cement on top of the skull. Animals had at least 7 days for recovery, and their body weights were checked daily.

\section{Operant Training}

The detailed procedures used for lever-press training are described elsewhere. ${ }^{46)}$ Briefly, animals were trained in an operant chamber $(30 \times 24 \times 24 \mathrm{~cm}$, model ENV-008VP; MED Associates, Fairfax, VT, USA). A customized program developed by LabView (National Instruments, Austin, TX, USA) was used to control the chamber through a digital input/output device (NI USB-6008; National Instruments) and to trigger the constant current stimulator (Model 2100; A-M Systems, Sequim, WA, USA) that delivered electrical stimulation to the animal. The biphasic electrical stimulation was characterized by a 300 ms pulse-train duration, $0.2 \mathrm{~ms}$ pulse-width, and a $4 \mathrm{~ms}$ inter-pulse interval $(250 \mathrm{~Hz})$.

After recovering from surgery, animals were initially shaped to press a lever in the chamber. After delivery of the free reward, animals voluntarily pressed the lever and electrical stimulation was contingently delivered to the MFB. Animals that showed abnormal behavior, such as jerking or head twitching, with stimulus delivery were not used. Stable lever-pressing performance was established two or three days after successful shaping. Rats that pressed the lever $>30$ presses per minute were used for subsequent experiments.

\section{Optimal Stimulus Intensity}

To find the optimal stimulus intensity, we constructed an intensity and response curve for each animal. A detailed procedure is described elsewhere. ${ }^{46)}$ Briefly, the number of lever presses performed by an animal was counted during a 3 minutes interval at an initial current intensity $(50 \mu \mathrm{A})$. The current intensity was increased by 25 $\mu \mathrm{A}$. As the intensity increased, animals pressed the lever more often, and the number of lever presses was counted at all intensity levels. When the number of lever presses was decreased or animals showed abnormal behavior (squeak and/or jerking), the procedure was stopped, and the reversal phase began (i.e., intensity decreases by 25 $\mu \mathrm{A}$ every 3 minutes). These processes were repeated twice. The total number of lever presses at each intensity level was examined. The optimal intensity level corresponding to the supramaximal response was chosen.
Once the current intensity was determined, it was fixed throughout the experiment.

Next, animals were ranked based on both the leverpress number at the optimal intensity level and body weights. Animals were then paired successively based on their ranking, and the rats in each group were randomly assigned either to the experimental training (E group) or the yoked control (C group).

\section{Experimental Design}

We performed theta suppression training for the theta band frequency $(4-8 \mathrm{~Hz})$. For the training, an animal pair ( $E$ and $C$ ) was individually placed in two identical and independently controlled operant chambers. Both animals' unilateral parietal cortex EEG were amplified (gain of 5,000) using an analog AC amplifier (7P511, Model 7 Polygraph; Grass Instruments, West Warwick, RI, USA) with a low-pass cut-off frequency of $100 \mathrm{~Hz}$. Amplified signals were then digitized by a data acquisition device (NI DAQPad-6015; National Instruments) with a sampling rate of $1 \mathrm{kHz}$. To compute the band amplitude of 4-8 $\mathrm{Hz}$, digital band-pass filtering (third order Butterworth) and the root-mean-square (RMS) value of the filtered signal were used. Band RMS was computed online for every $50 \mathrm{~ms}$ bin.

A single daily session of suppression training consisted of three consecutive blocks (10 minutes of pre-training, PRE; 30 minutes of suppression training, TRAIN; and 10 minutes of post-training, POST) (Fig. 1A). In the PRE block, the EEG was recorded, and the band RMS of 4 to 8 $\mathrm{Hz}$ was computed. At the end of the PRE block, the band RMS values of the $E$ rat were averaged and $50 \%$ of the computed average was used as a threshold level for the TRAIN block.

During the TRAIN block, the band RMS amplitude of each $250 \mathrm{~ms}$ bin was compared with the threshold. Whenever the band RMS value was less than the determined threshold level, animals of both groups received reward MFB stimulation (Fig. 1B). Therefore, reward stimulation was delivered contingently to the animals in the $\mathrm{E}$ group, and non-contingent stimuli were delivered to the animals in the $\mathrm{C}$ group (Fig. 1C). To avoid continuous reward stimulation, which may produce convulsions, animals were not allowed to receive another reward stimulus within $500 \mathrm{~ms}$ following previous stimulation.

Animals were trained for ten sessions over two weeks. 
A

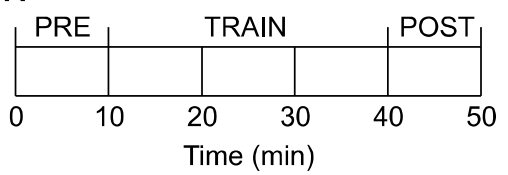

B

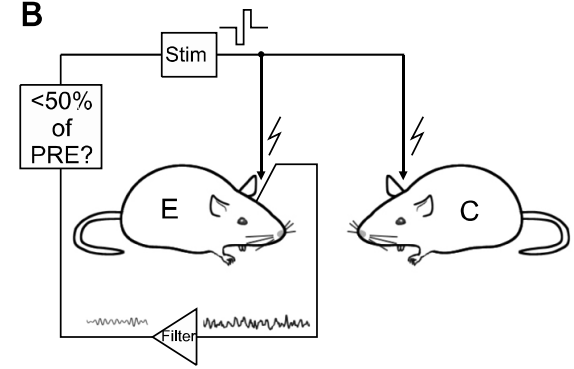

C

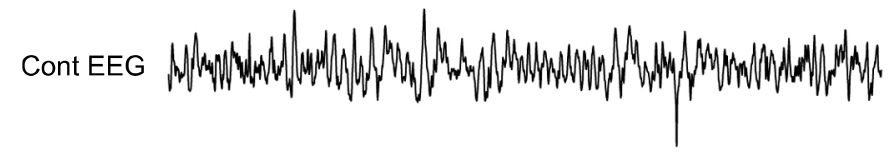

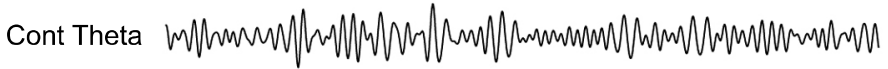

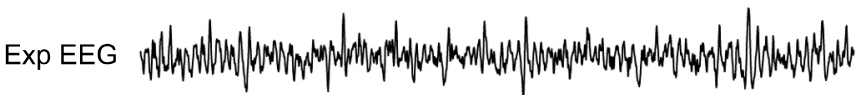

Fig. 1. Experimental setup and contingent reward delivery. (A) A single daily session consisted of three blocks: PRE (10 minutes of pre-training), TRAIN (30 minutes of suppression training), and POST (10 minutes of post-training). (B) During TRAIN block, the parietal electroencephalography (EEG) of the experimental group (E group) rats was band-pass filtered, and the band root-mean-square (RMS) value of each $250 \mathrm{~ms}$ bin was calculated. Whenever the band RMS value was less than the determined threshold level, the reward (medial forebrain bundle stimulation) was delivered to both the experimental (Exp) and control (Cont) rats. (C) Example traces of parietal EEGs from E and C group rats. The arrowheads indicate the reward stimulation delivered during training.

Throughout the sessions, EEGs, band amplitudes, and rewarding time were stored on a computer for later offline analyses.

\section{Analysis}

From the reward MFB stimulation records, the number of stimuli over the 5 minutes bin of the TRAIN block were calculated.

Recorded EEGs were analyzed offline using customized programs developed by Matlab (MathWorks, Natick, MA, USA). The band RMS values were computed using the digital Butterworth filter (the same as used for online training). The band RMS values were averaged for every 5 minutes bin.

EEG amplitude spectrums for each block were assessed. The Hanning window was multiplied by the raw EEG for a given block, and the EEG spectrum was computed using the Fast Fourier Transform algorithm. Binning and moving average algorithms were further applied to the spectrum for smoothing. The frequency resolution of the resulting spectrum was $0.05 \mathrm{~Hz}$. Spectrums for the PRE $(10 \mathrm{mi}-$ nutes) block, the last 10 minutes of the TRAIN block, and the POST (10 minutes) block were assessed.

The spectral theta peak was defined as the peak within the frequency range of 4 to $10 \mathrm{~Hz}$, and its frequency and amplitude were calculated.
Stimulus-triggered averages of EEG signals and bandpass filtered signals were calculated from signals 1 second before to 2 seconds after the beginning of stimulation for each animal. Then, the RMS amplitudes for 4 periods $(-1$ to -0.25 second, -0.25 to 0 second, 0 to 1 second, and 1 to 2 seconds of stimulation) of the stimulus-triggered average were calculated.

\section{Statistical Analysis}

Three-way ANOVA was used to compare the differences in theta peak frequency and amplitude between groups, sessions, and blocks. Then, one-way ANOVA with Tukey's post hoc analysis was used to compare the differences at each block. To compare spectral profiles, $t$ tests were used to test differences of amplitude in each frequency bin.

Two-way repeated measures ANOVA was used to compare the differences between groups over time. One-way ANOVA with Tukey's post hoc analysis was also used. Two-tailed t tests were also used to test the mean differences of specific data between the two groups. All data were represented in the form of mean \pm standard error of mean. The $p$ values smaller than 0.05 were considered significant except in comparisons of spectral profiles in which $p$ values smaller than 0.01 were considered significant. Matlab Statistical Toolbox (MathWorks, 
Natick, MA, USA) was used for statistical analysis.

\section{RESULTS}

\section{Lever-press Training}

Animals that pressed the lever less than 30 times per minute or showed abnormal responses to the MFB stimulation during lever-press training were excluded in this study. Otherwise, we deemed that the implantation surgery was successful, and electrode tips were correctly positioned at the MFB area. A total of 16 rats showed stable lever-press performance and were used for theta suppression training. Eight pairs of rats were assigned to either a yoked control (C) or an experimental (E) group. The mean current intensity of the MFB stimulation was $116.67 \pm 34.16 \mu \mathrm{A}$ (mean \pm standard deviation).

\section{Reward Stimuli-evoked Cortical Activity}

The stimulation-evoked cortical activity in both groups increased in amplitude. The stimulus-triggered EEG average and filtered signal of both groups are shown in Figure $2 \mathrm{~A}$ and $2 \mathrm{~B}$, respectively. Raw EEG and theta activity were aligned by stimulus time. This alignment shows the effect of the electrical stimulation on the cortical EEG. Root mean square (RMS) amplitudes of 4 periods around the stimulus-triggered theta activity are depicted in Figure 2C. RMS values in the $\mathrm{E}$ group were lower than in the $\mathrm{C}$ group $(\mathrm{F}(1,42)=4.73, p=0.047)$, and RMS values in both groups were different among periods $(F(3,42)=36.81, p=0.000)$, but there was no interaction between groups and periods $(\mathrm{F}(3,42)=1.81, p=0.160)$. RMS amplitudes of the first and second periods $(-1.0$ to -0.25 second and -0.25 to 0 second) ( $p=0.0314$ and 0.000 , respectively) before stimulus were lower in group $\mathrm{E}$ than in group $\mathrm{C}$. The stimulus evoked an approximately $6 \mathrm{~Hz}$ rhythm locked to the stimulus.

\section{Number of Reward Stimuli}

The number of reward stimuli during feedback training is shown in Figure 3. It gradually increased along with the successive 5 minutes bins of the training block (withinsession) in the first $(F(5,35)=2.63, p=0.041)$ and tenth session $(F(5,35)=8.55, p=0.000)$ and in the pooled data (Fig. $3 \mathrm{~A} ; \mathrm{F}(5,35)=8.66, p=0.000)$. The number of reward stim-

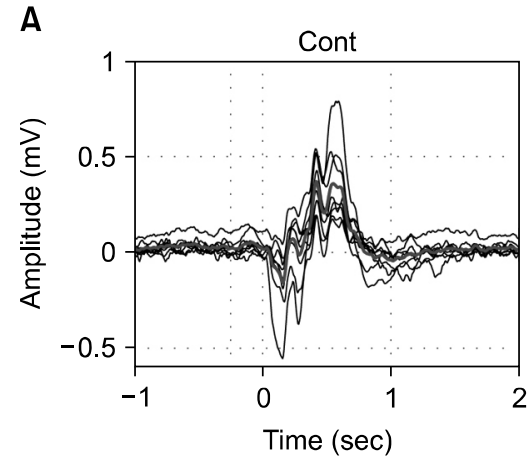

B

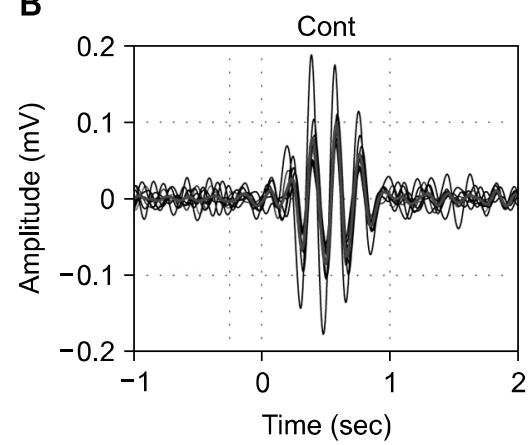

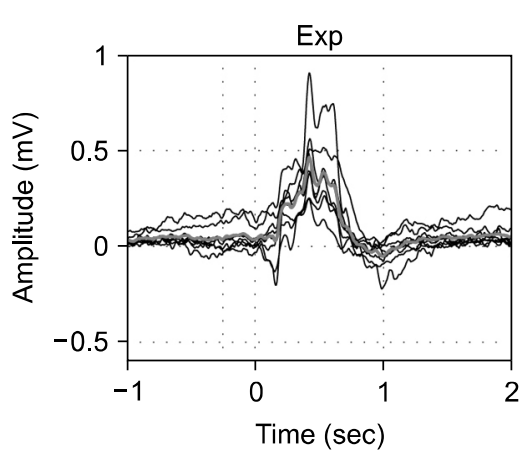

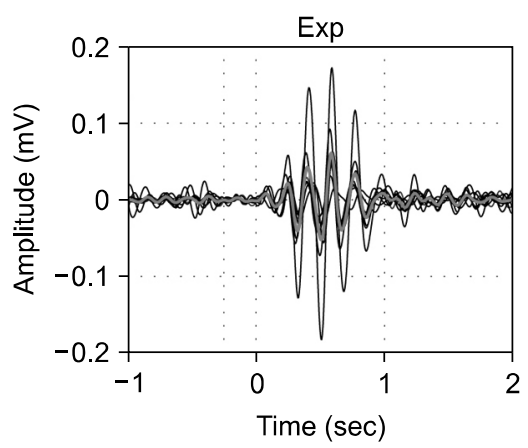

C

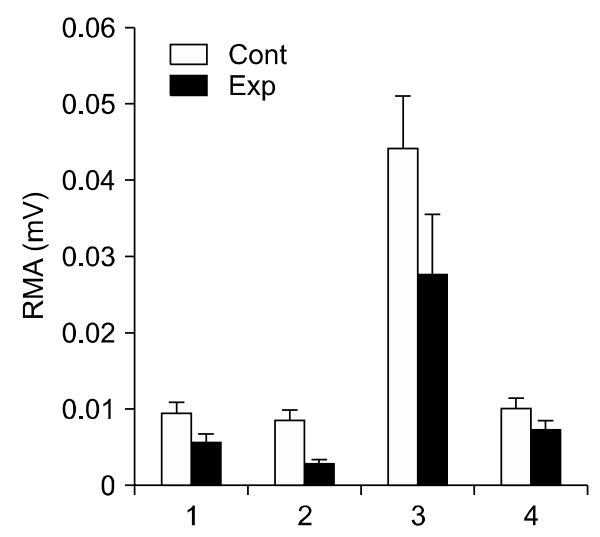

Fig. 2. The stimulus triggered average of electroencephalography $(\mathrm{A})$ and band-pass $(4-8 \mathrm{~Hz})$ filtered signals $(\mathrm{B})$ during the training block. Electrical stimulation induced time-locked $6 \mathrm{~Hz}$ oscillations with raised amplitudes. (C) Root-mean-square amplitudes of periods 1 second before and after the stimulus.

Cont, control; Exp, experimental. 

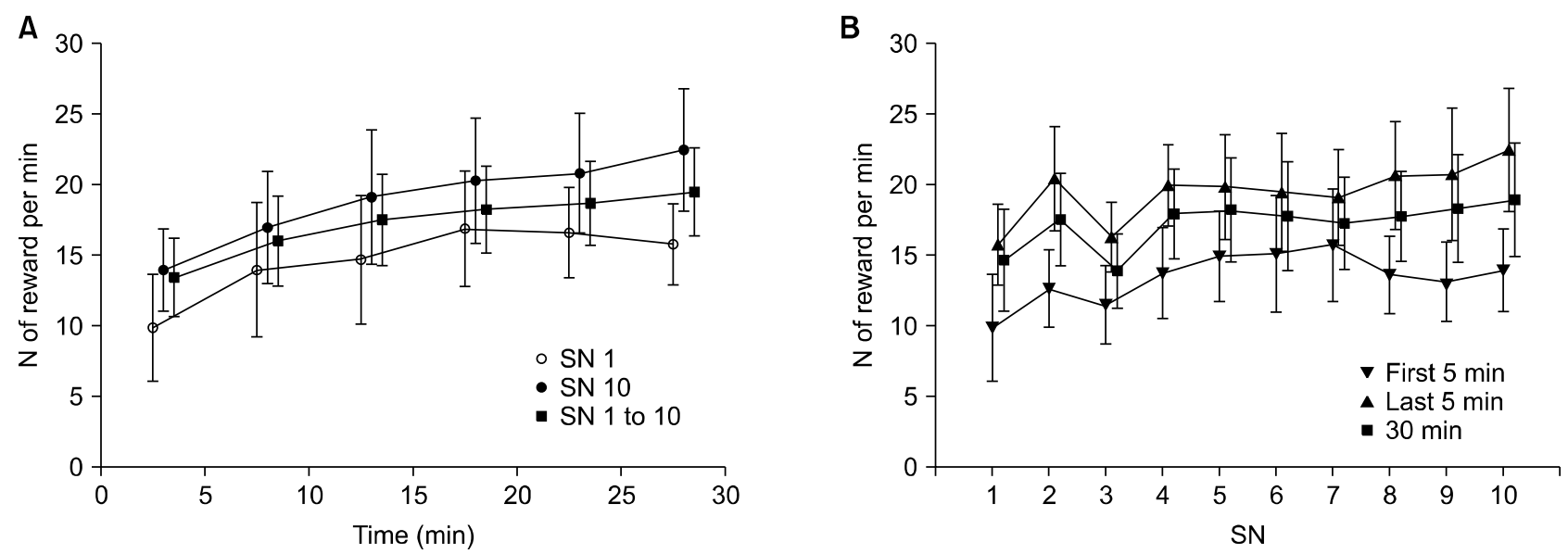

Fig. 3. (A) Number of rewards received in successive 5 minutes bins during the TRAIN block. Rewards tended to significantly increase with time $(\mathrm{F}(5,35)=2.62, p=0.041$ in the first session; $\mathrm{F}(5,35)=8.55, p=0.000$ in the 10 th session; and $\mathrm{F}(5,35)=8.66, p=0.000$ in all pooled sessions). (B) The number of rewards within the sessions increased significantly $(\mathrm{F}(9,63)=2.73, p=0.009$ in the first 5 minutes; $\mathrm{F}(9,63)=2.61, p=0.012$ in the last 5 minutes; and $\mathrm{F}(9,63)=2.88, p=0.007$ in the whole block).

$\mathrm{SN}$, session number.

uli gradually increased across sessions (Fig. 3B; $F(9,63)=$ 2.88, $p=0.007$ ). This indicates that subjects could learn to suppress theta activity gradually with operant conditioning training.

\section{Changes in Spectral Profiles}

Spectral profiles of the parietal cortex EEG were examined by comparing the amplitude of each frequency bin using t tests. First, the amplitudes were compared between blocks (Fig. 4A). During the first session, the amplitudes of most frequency bins in the training band $(4-8 \mathrm{~Hz})$ decreased during the TRAIN block compared to the PRE block in both the $\mathrm{C}$ and $\mathrm{E}$ groups. There was also increased amplitude in most frequency bins in the higher frequency bands (alpha band, 8-12 Hz) in the TRAIN block compared to the PRE block. During the tenth session, the training band and higher frequency amplitudes were significantly different only in the $\mathrm{E}$ group.

The amplitudes were compared between the first session and the last session (Fig. 4B). No significant differences were observed between sessions in the $C$ group, while the amplitudes of a few frequency bins in the theta band and the alpha band were different in the $\mathrm{E}$ group.

The amplitudes were compared between groups $C$ and $\mathrm{E}$ (Fig. 4C). Amplitudes of only a few frequency bins were different between groups for the first session, but the amplitudes of most frequency bins in the theta band and the alpha band were different between groups. This result in- dicates that the amplitude differences between groups grew with successive sessions.

The shape of the spectral profiles showed that the spectral theta peak shifted to the higher frequency range more in the $\mathrm{E}$ group than in the $\mathrm{C}$ group with suppression training. We then examined the peak amplitude and frequency of the spectrum in the first and tenth sessions (Fig. 4D). The frequency of the spectral peak was significantly different between groups $(\mathrm{F}(1,85)=22.95, p=0.000)$ and between sessions $(F(1,85)=8.97, p=0.004)$ and among the three blocks $(\mathrm{F}(2,85)=38.01, p=0.000)$. The amplitude of the spectral peak was not different between groups $(F(1,85)=0.38, p=0.539)$, but was significantly different between sessions $(\mathrm{F}(1,85)=5.5, p=0.021)$ and among the three blocks $(\mathrm{F}(2,85)=13.14, p=0.000)$.

These results indicate that the decrease of the amplitude of the theta band is accompanied by the gradual shift of the frequency of the theta peak through sessions of theta suppression training, and the decrease of the amplitude of the theta band and the shift of the frequency shift are maintained after the training sessions (Fig. 4).

\section{Changes in Band RMS Amplitudes}

The changes in theta activity, the suppression training target, within a session and across sessions are shown in Figure $5 \mathrm{~A}$ and $5 \mathrm{~B}$, respectively. Theta activity tended to decrease in the $E$ group with no changes in the $C$ group within the TRAIN block (Fig. 5A). Training decreased the- 

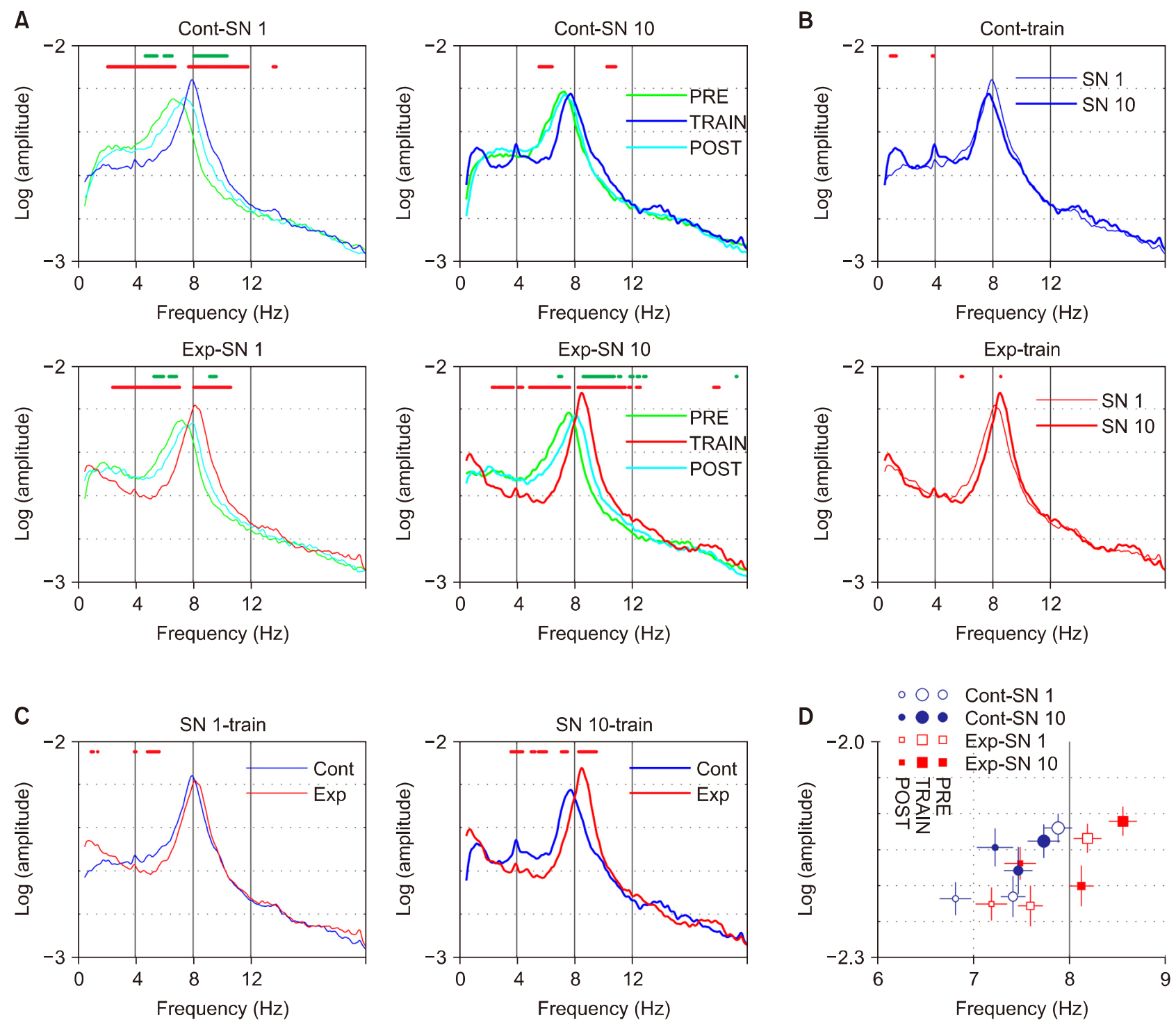

Fig. 4. Spectral profiles of parietal cortex electroencephalography. (A) Among periods (PRE, TRAIN and POST), (B) between groups (Cont and Exp), and (C) between the first and last sessions (SN 1 and SN 10). Significant differences are indicated with dots on the plot ( $p<0.01$ ). (D) Plot of frequency and amplitude of theta peaks at each period before (PRE), during (TRAIN), and after (POST) conditioning training. Significant differences are indicated with asterisk $(p<0.05)$.

PRE, 10 minutes of pre-training; TRAIN, 30 minutes of suppression training; POST, 10 minutes of post-training; Cont, control; Exp, experimental; SN, session number.

ta activity compared to the pre-training level in the $\mathrm{E}$ group $(F(1,14)=21.168, p=0.000)$ with no differences between the first session and the tenth session $(\mathrm{F}(1,14)=0.005, p=0.946)$. In the $\mathrm{C}$ group, theta activity did not change within the TRAIN block, but there was a slight increase during the TRAIN block for the tenth session. In the POST block, theta activity was restored to its pre-training levels in both groups (Fig. 5A). The theta activity for the last bin of the TRAIN block did not sig- nificantly change across sessions $(F(9,126)=1.583$, $p=0.127)$, but it was different between groups $(F(1,14)=$ 10.418, $p=0.006$ ) (Fig. 5B).

There were no significant differences between groups in other bands (data not shown) except the alpha band. As shown in Figure 5C, alpha activity tended to increase during the TRAIN block in both groups (C group: $F(1,14)=$ 43.877, $p=0.000$; E group: $\mathrm{F}(1,14)=57.783, p=0.000$ ), and there were no differences between the first and tenth 

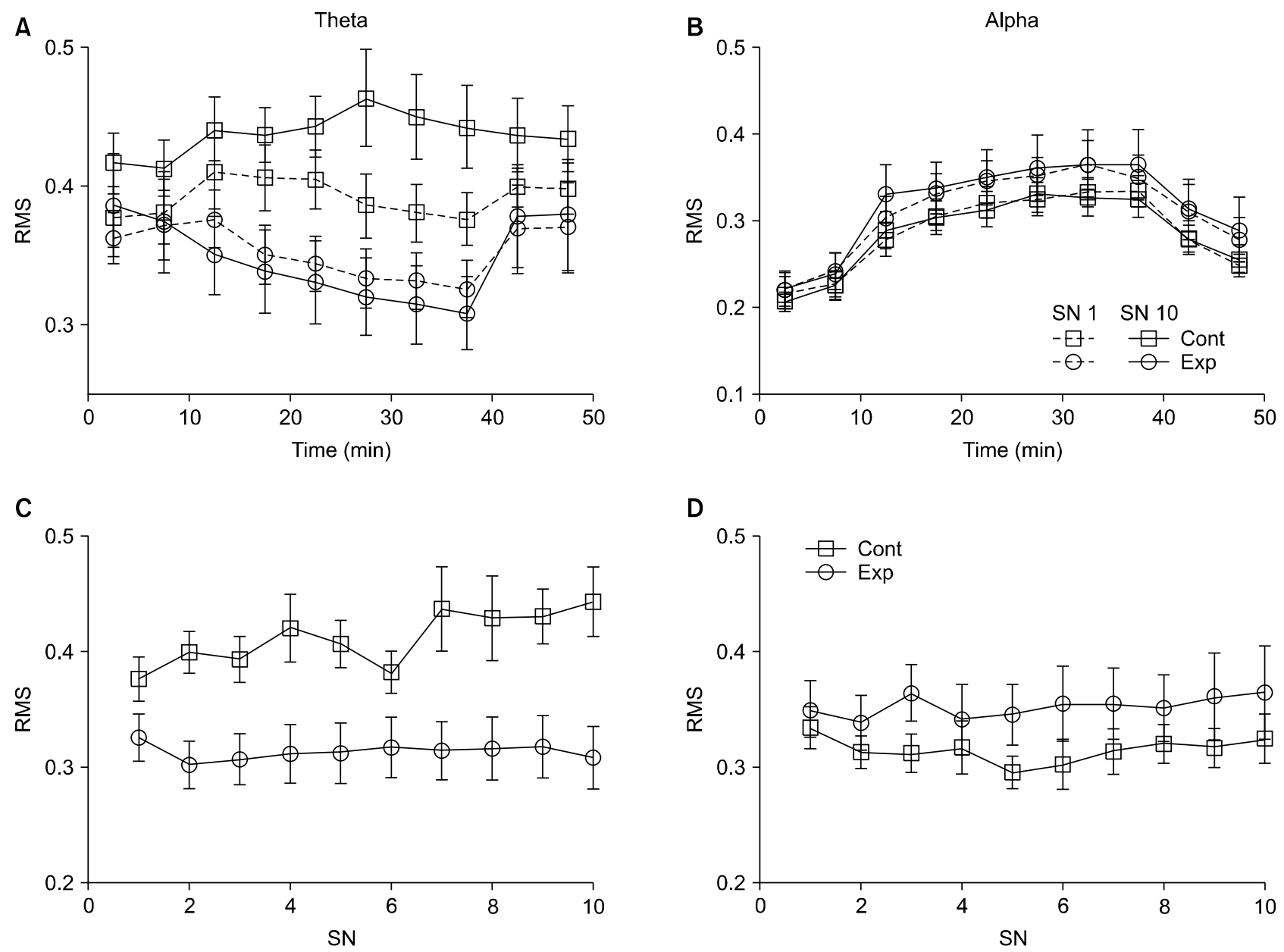

Fig. 5. Changes in theta (left column) and alpha (right column) activity (root-mean-square [RMS]). (A, B) Intra-session changes in the first and tenth sessions. Note the theta activity decreased over time in the TRAIN period in the experimental group but not in the control group. Alpha activity increased over time during the TRAIN period in both groups. (C, D) Activity changes in the TRAIN period across sessions. Theta activity remained unchanged (or slightly increased) in the control group but was significantly lower in the experimental group $(p<0.05)$. Alpha activity remained unchanged with no differences between groups.

PRE, 10 minutes of pre-training; TRAIN, 30 minutes of suppression training; POST, 10 minutes of post-training; Cont, control; Exp, experimental; SN, session number.

sessions $(\mathrm{F}(1,14)=1.379, p=0.260)$. The alpha activity for the last bin of the TRAIN block did not significantly change across sessions and was not significantly different between groups (Fig. 5D).

\section{DISCUSSION}

In this study, we examined the spectral profile changes in a neurofeedback animal model using intracranial electrical stimulation to the MFB as a reward and whether the animal could voluntarily control its brain activity, specifically theta activity suppression in this study. Training of theta activity suppression gradually occurred in the $\mathrm{E}$ group but not in the $\mathrm{C}$ group within sessions and across sessions. It seemed that rats could voluntarily control their brain activity, and this training could be accomplished through operant conditioning.

The ICSS-operant conditioning paradigm could be used to study practical training of self-control of brain activity. However, there are some restrictions. First, electrical reward can elicit transient oscillations artificially in the brain, which are undesirable. ${ }^{45)}$ This effect makes it somewhat unmanageable to examine the exact quantity of brain activity modulated voluntarily. Second, rats can show rearing and sniffing behavior when they are rewarded with electrical stimulation. ${ }^{47)}$ To avoid con- 
tamination due to electrical stimulation-induced theta activity, we instead tested suppression of theta activity.

The aim of this study was to suppress band activity within the theta frequency range. We used electrical stimulation delivered to the MFB as a reward whenever the experimental rat suppressed its theta activity. The MFB is widely understood for its function in reward and motivation. ${ }^{40,42,48,49)}$ Animals were allowed to move freely during training sessions. When stimulation was delivered, highly increased locomotion as well as searching behavior was observed (actually we only measured two pairs of rats), as previously described. ${ }^{47)}$ Since both the speed of locomotion and the motivational state may affect theta rhythm frequency, ${ }^{43)}$ the observed shift of the theta peak might be explained by the modulation effect of locomotion and not by MFB stimulation. In fact, the frequency of the theta rhythm-evoked response by MFB stimulation was around $6 \mathrm{~Hz}$, so the shift of the theta peak to the higher frequency might be explained by increases in locomotion rather than MFB stimulation even though overall theta activity was decreased in the experimental group but not in the control group.

Theta activity was reduced significantly within the training period of a single session. The reduced theta activity was hardly maintained in the subsequent sessions. This result is in line with human alpha neurofeedback training. ${ }^{20)}$ One possibility for this finding is that we only had 10 sessions of feedback training, which does not seem to be enough to constantly alter brain activity. ${ }^{32)}$ Nonetheless, theta activity gradually increased across sessions.

Theta reduction in the $\mathrm{E}$ group compared to the $\mathrm{C}$ group was a specific change in response to suppression training. The activities of other bands except the alpha band were not changed significantly during sessions and across sessions. However, theta suppression was not maintained across sessions in the E group; rather, theta activity in the $C$ group increased across sessions. Alpha activity increased during training in both groups. The relatively suppressed theta in the $\mathrm{E}$ group compared to the $\mathrm{C}$ group was due to the shift of the theta peak frequency during theta suppression training. This shift observed during the training block might be explained by a dependency of the theta rhythm on locomotor speed and the motivational-emotional state. ${ }^{43)}$ This property might have maintained low theta activity in the E group but increased alpha activity in both groups. In the $\mathrm{C}$ group, reward stimulation might have caused nonspecific locomotion and theta activity.

It appears that electrical reward itself affected the modulation of the spectral peak. It has been reported that electrical stimulation enhances the $8-12 \mathrm{~Hz}$ (referred to as alpha) activity. ${ }^{45)}$ However, the theta peak moved above 8 $\mathrm{Hz}$ gradually only in the $\mathrm{E}$ group but not in the $\mathrm{C}$ group, so the reward stimulation may not have caused the theta peak shift. Instead, this suppression training may cause a shift of the resonance frequency of cortical theta oscillations.

For the spectral peak shift, a resonance network model has been proposed. ${ }^{50)}$ We confirmed that the band activity was practically affected by the spectral peak, which represents the dominant oscillation frequency resulting from the synchronization of neuronal population. Therefore, how an individual can modulate his or her brain activity voluntarily encompasses the question of how oscillatory networks generate resonance frequencies (or an “optimal coupling level," according to Lubar ${ }^{50)}$ ).

Most human neurofeedback training has been focused on either specific band power regulation ${ }^{16-18,32)}$ or the frequency of the alpha peak. ${ }^{51)}$ It is still controversial whether the spectral profile changes during neurofeedback training. ${ }^{31)}$ Our results demonstrated that spectral changes were clearly shown during training but disappeared a few minutes after training. However, there was a remaining effect in the next training session. This finding suggestsa learning effect of theta suppression. It might be preferable to assess EEG spectraduring neurofeedback training. ${ }^{52)}$

Current neurofeedback training focuses on the control of specific band power. Our data suggests that band power is not independently modulated. Rather, it is closely related to its neighboring bands and to the shift of the spectral peak and the modulation of the resonant frequency span over these bands. There are a large number of clinical studies that use the alpha/theta ratio as a feedback parameter. ${ }^{32,53)}$ This might be a result of the spectral peak shift from theta to alpha and vice versa.

When a spectral peak is located in or near the band to be regulated, overall spectral dynamics (peak amplitude and frequency) seem to play a crucial role. In such a case, band activity regulation is highly correlated with peak-associated modulation, not a uniform suppression over the whole range of the training band. This might also influence the level of training difficulty. Therefore, the exami- 
nation of overall spectral profiles during training should be required for better neurofeedback training.

We proposed a new framework to study neurofeedback using animals. Incorporating the ICSS paradigm, it allowed rats to control their brain activity according to experimental criteria. Furthermore, this setup gave us the possibility to investigate the mechanism of voluntary EEG modulation. Spectral shift appears to be one of the primary processes involved in band activity regulation together with resonance networks. This operant conditioning of the animal EEG will lead to new insights into the mechanism of neurofeedback as well as oscillatory modulations in the brain.

It is unclear whether neurofeedback training can be considered operant conditioning of specific band activity. It is thought that in neurofeedback a subject can voluntarily control brain activity through awareness of the brain activity state with a perceivable physical stimulus representing that activity and through the expectation that the consequence of the change of brain activity will result in a reward; this idea contrasts operant conditioning in which control of a voluntary motor behavior is naturally perceivable. Non-perceivable functional changes, such as brain activity or involuntary autonomic physiological changes, are trained in neurofeedback or biofeedback, ${ }^{22)}$ while a perceivable behavior like a lever-press is trained in operant or instrumental conditioning. ${ }^{40)}$ We supposed that physical stimuli representing brain activity instead of wanted brain activity could be perceived and potentiated, and then the status producing specific brain activity may be conditioned during neurofeedback training.

Our results lead to the question of how animals achieve their goal and what strategies they use. These subtle changes of physiological activity are not consciously explicitly perceived and controlled, but it is suggested that a certain specific strategy could be used to control the subtle changes. ${ }^{39)}$ In fact, autonomic functions cannot be controlled directly but indirectly, and direct control of autonomic functions remains to be demonstrated. ${ }^{54)}$ EEG biofeedback is only one exception that is suggested to be operant conditioned. ${ }^{55)}$

There is a difference between operant conditioning and human neurofeedback. There is no explicit contingent reward in neurofeedback training in humans, while there is an explicit contingent reward in operant conditioning. Our results indicated that learning how to reduce band activity was rapidly completed in the first session, maybe due to the reward modality. ${ }^{49,56)}$ This result is not consistent in human neurofeedback studies. For example, operant conditioning of the lever-press behavior gradually increased within sessions and across sessions, while control of brain activity in human has been reported differently from our results. Theta/alpha feedback training increases the rhythm ratio within sessions but not across sessions, ${ }^{32)}$ and theta activity increases along sessions. ${ }^{57)}$ In contrast, alpha activity gradually decreases within sessions, but baseline alpha increases along sessions in alpha feedback. ${ }^{20,52)}$ Sometimes, neighboring band activity changes accompany target band feedback training, such as changes in alpha activity during low beta neurofeedback. ${ }^{41)}$ Collectively, neurofeedback training did not seem to gradually change the training target, while, in contrast, behavioral training gradually increased in operant conditioning.

To develop a neurofeedback animal model, we used operant conditioning of brain activity using ICSS to the MFB as a reward. Rats could learn to suppress band activity across sessions through changes of the spectral profile accompanied by peak frequency shift rather than actual suppression of specific brain activity. Our results showed similar characteristics reported in human neurofeedback studies. Our animal model could be used as a model for human neurofeedback.

\section{- Acknowledgments}

We thank Dr. Pyung Choi, Dr. J. J. Chrobak and Dr. C. T. Unal for commenting on an early draft of this paper, and we thank Ji Young Jung and Minji Kim for technical assistance. This work was supported by the Basic Science Research Program through the NRF funded by the Ministry of Education, Science and Technology (20100022362, 2012R1A2A2A02046812), and by a fund of the Hankok Medical Science Foundation (2009).

\section{REFERENCES}

1. Klimesch W. EEG alpha and theta oscillations reflect cognitive and memory performance: a review and analysis. Brain Res Brain Res Rev 1999;29:169-195.

2. Buzsáki G, Draguhn A. Neuronal oscillations in cortical networks. Science 2004;304:1926-1929.

3. Başar E, Güntekin B. A review of brain oscillations in cognitive disorders and the role of neurotransmitters. Brain Res 2008;1235:172-193. 
4. Düzel E, Penny WD, Burgess N. Brain oscillations and memory. Curr Opin Neurobiol 2010;20:143-149.

5. Mizumori SJ, Perez GM, Alvarado MC, Barnes CA, McNaughton BL. Reversible inactivation of the medial septum differentially affects two forms of learning in rats. Brain Res 1990;528:12-20.

6. Bland $\mathrm{BH}$, Oddie SD. Theta band oscillation and synchrony in the hippocampal formation and associated structures: the case for its role in sensorimotor integration. Behav Brain Res 2001;127:119-136.

7. Seager MA, Johnson LD, Chabot ES, Asaka Y, Berry SD. Oscillatory brain states and learning: Impact of hippocampal theta-contingent training. Proc Natl Acad Sci U S A 2002;99: 1616-1620.

8. Griffin AL, Asaka Y, Darling RD, Berry SD. Theta-contingent trial presentation accelerates learning rate and enhances hippocampal plasticity during trace eyeblink conditioning. Behav Neurosci 2004;118:403-411.

9. Caplan JB, Madsen JR, Schulze-Bonhage A, AschenbrennerScheibe R, Newman EL, Kahana MJ. Human theta oscillations related to sensorimotor integration and spatial learning. I Neurosci 2003;23:4726-4736.

10. Uhlhaas PJ, Singer W. Neural synchrony in brain disorders: relevance for cognitive dysfunctions and pathophysiology. Neuron 2006;52:155-168.

11. Winson J. Loss of hippocampal theta rhythm results in spatial memory deficit in the rat. Science 1978;201:160-163.

12. McNaughton N, Ruan M, Woodnorth MA. Restoring theta-like rhythmicity in rats restores initial learning in the Morris water maze. Hippocampus 2006;16:1102-1110.

13. Sterman MB. Effects of brain surgery and EEG operant conditioning on seizure latency following monomethylhydrazine intoxication in the cat. Exp Neurol 1976;50:757-765.

14. Beatty J, Greenberg A, Deibler WP, O'Hanlon JF. Operant control of occipital theta rhythm affects performance in a radar monitoring task. Science 1974;183:871-873.

15. Nan W, Rodrigues JP, Ma J, Qu X, Wan F, Mak PI, et al. Individual alpha neurofeedback training effect on short term memory. Int I Psychophysiol 2012;86:83-87.

16. Zoefel B, Huster RJ, Herrmann CS. Neurofeedback training of the upper alpha frequency band in EEG improves cognitive performance. Neuroimage 2011;54:1427-1431.

17. Ros T, Moseley MJ, Bloom PA, Benjamin L, Parkinson LA, Gruzelier $\mathrm{JH}$. Optimizing microsurgical skills with EEG neurofeedback. BMC Neurosci 2009;10:87.

18. Hoedlmoser K, Pecherstorfer T, Gruber G, Anderer P, Doppelmayr M, Klimesch W, et al. Instrumental conditioning of human sensorimotor rhythm $(12-15 \mathrm{~Hz})$ and its impact on sleep as well as declarative learning. Sleep 2008;31:14011408.

19. Hermer-Vazquez L, Hermer-Vazquez R, Rybinnik I, Greebel G, Keller R, Xu S, et al. Rapid learning and flexible memory in "habit" tasks in rats trained with brain stimulation reward.
Physiol Behav 2005;84:753-759.

20. Cho MK, Jang HS, Jeong SH, Jang IS, Choi BJ, Lee MG. Alpha neurofeedback improves the maintaining ability of alpha activity. Neuroreport 2008;19:315-317.

21. Hardt JV, Kamiya J. Anxiety change through electroencephalographic alpha feedback seen only in high anxiety subjects. Science 1978;201:79-81.

22. Sterman MB, Egner T. Foundation and practice of neurofeedback for the treatment of epilepsy. App/ Psychophysiol Biofeedback 2006;31:21-35.

23. Monderer RS, Harrison DM, Haut SR. Neurofeedback and epilepsy. Epilepsy Behav 2002;3:214-218.

24. Lubar JF, Swartwood MO, Swartwood JN, O'Donnell PH. Evaluation of the effectiveness of EEG neurofeedback training for $A D H D$ in a clinical setting as measured by changes in T.O.V.A. scores, behavioral ratings, and WISC-R performance. Biofeedback Self Regul 1995;20:83-99.

25. Fox DJ, Tharp DF, Fox LC. Neurofeedback: an alternative and efficacious treatment for Attention Deficit Hyperactivity Disorder. Appl Psychophysiol Biofeedback 2005;30:365373.

26. Saxby E, Peniston EG. Alpha-theta brainwave neurofeedback training: an effective treatment for male and female alcoholics with depressive symptoms. J Clin Psychol 1995;51:685-693.

27. Peniston EG, Kulkosky PJ. Alpha-theta brainwave training and beta-endorphin levels in alcoholics. Alcohol Clin Exp Res 1989;13:271-279.

28. Peniston EG, Kulkosky PJ. Alpha-theta brainwave neuro-feedback therapy for Vietnam veterans with combat-related post-traumatic stress disorder. Med Psychother 1991;4:4760.

29. Hammond DC. Neurofeedback treatment of depression and anxiety. J Adult Dev 2005;12:131-137.

30. Vernon D, Egner T, Cooper N, Compton T, Neilands C, Sheri $\mathrm{A}$, et al. The effect of training distinct neurofeedback protocols on aspects of cognitive performance. Int I Psychophysiol 2003:47:75-85.

31. Vernon DJ. Can neurofeedback training enhance performance? An evaluation of the evidence with implications for future research. Appl Psychophysiol Biofeedback 2005;30: 347-364.

32. Raymond J, Sajid I, Parkinson LA, Gruzelier JH. Biofeedback and dance performance: a preliminary investigation. Appl Psychophysiol Biofeedback 2005;30:64-73.

33. Boulay CB, Sarnacki WA, Wolpaw JR, McFarland DJ. Trained modulation of sensorimotor rhythms can affect reaction time. Clin Neurophysiol 2011;122:1820-1826.

34. Wyrwicka W, Sterman MB. Instrumental conditioning of sensorimotor cortex EEG spindlesin the waking cat. Physiol Behav 1968:3:703-707.

35. Gruzelier J, Egner T, Vernon D. Validating the efficacy of neurofeedback for optimising performance. Prog Brain Res 2006; 159:421-431. 
36. Watson CG, Herder J. Effectiveness of alpha biofeedback therapy: negative results. J Clin Psychol 1980;36:508-513.

37. Enck P, Benedetti F, Schedlowski M. New insights into the placebo and nocebo responses. Neuron 2008;59:195-206.

38. Williams P. E.E.G. alpha feedback--a comparison of two control groups. Psychosom Med 1977;39:44-47.

39. Cott A, Pavloski RP, Goldman JA. Cortical alpha rhythm, biofeedback, and the determinants of subjective state. J Exp Psychol Gen 1981;110:381-397.

40. Olds J, Milner P. Positive reinforcement produced by electrical stimulation of septal area and other regions of rat brain. J Comp Physiol Psychol 1954;47:419-427.

41. Egner T, Gruzelier JH. EEG biofeedback of low beta band components: frequency-specific effects on variables of attention and event-related brain potentials. Clin Neurophysiol 2004;115:131-139.

42. Carlezon WA Jr, Chartoff EH. Intracranial self-stimulation (ICSS) in rodents to study the neurobiology of motivation. Nat Protoc 2007;2:2987-2995.

43. Sławińska U, Kasicki S. The frequency of rat's hippocampal theta rhythm is related to the speed of locomotion. Brain Res 1998;796:327-331.

44. Shankaranarayana Rao BS, Raju TR, Meti BL. Self-stimulation of lateral hypothalamus and ventral tegmentum increases the levels of noradrenaline, dopamine, glutamate, and AChE activity, but not 5-hydroxytryptamine and GABA levels in hippocampus and motor cortex. Neurochem Res 1998;23:10531059.

45. Latash LP, Kovalzon VM. LHA self-stimulation effects on EEG and brain temperature in white rats. Physiol Behav 1973;10: 651-655.

46. Lee MG, Jun G, Choi HS, Jang HS, Bae YC, Suk K, et al. Operant conditioning of rat navigation using electrical stimulation for directional cues and rewards. Behav Processes 2010;84:715-720.

47. Ikemoto S, Panksepp J. The relationship between self-stim- ulation and sniffing in rats: does a common brain system mediate these behaviors? Behav Brain Res 1994;61:143-162.

48. Wise RA. Dopamine, learning and motivation. Nat Rev Neurosci 2004;5:483-494.

49. Olds ME, Fobes JL. The central basis of motivation: intracranial self-stimulation studies. Annu Rev Psychol 1981;32: 523-574.

50. Lubar JF. Neocortical dynamics: implications for understanding the role of neurofeedback and related techniques for the enhancement of attention. Appl Psychophysiol Biofeedback 1997;22:111-126.

51. Angelakis E, Stathopoulou S, Frymiare JL, Green DL, Lubar JF, Kounios J. EEG neurofeedback: a brief overview and an example of peak alpha frequency training for cognitive enhancement in the elderly. Clin Neuropsychol 2007;21:110-129.

52. Dempster T, Vernon D. Identifying indices of learning for alpha neurofeedback training. Appl Psychophysiol Biofeedback 2009;34:309-328.

53. Fernández T, Herrera W, Harmony T, Díaz-Comas L, Santiago $\mathrm{E}$, Sánchez L, et al. EEG and behavioral changes following neurofeedback treatment in learning disabled children. Clin Electroencephalogr 2003;34:145-152.

54. Dworkin BR, Miller NE. Failure to replicate visceral learning in the acute curarized rat preparation. Behav Neurosci 1986; 100:299-314.

55. Birbaumer N, Cohen LG. Brain-computer interfaces: communication and restoration of movement in paralysis. J Physiol 2007;579:621-636.

56. Ruiz-Medina J, Redolar-Ripoll D, Morgado-Bernal I, AldavertVera L, Segura-Torres P. Intracranial self-stimulation improves memory consolidation in rats with little training. Neurobiol Learn Mem 2008:89:574-581.

57. Egner T, Gruzelier JH. Ecological validity of neurofeedback: modulation of slow wave EEG enhances musical performance. Neuroreport 2003;14:1221-1224. 